\title{
IEEE 802.11 DCF performance evaluation using one-dimensional discrete time chains
}

\author{
Srikant Kuppa ${ }^{\dagger}$, Shun-Chen Niu ${ }^{\dagger}$ and Ravi Prakash ${ }^{\dagger}$ \\ $\dagger$ Department of Computer Science \\ $\ddagger$ School of Management \\ The University of Texas at Dallas \\ Richardson, TX 75083 \\ Email: \{ksrikant, scniu, ravip\}@utdallas.edu
}

\begin{abstract}
Previously proposed analytical studies for IEEE 802.11 Distributed Coordination Function model the behavior of a wireless station operating under saturation conditions as a twodimensional discrete time Markov chain. In this document, we show that the two-dimensional chain can be partitioned into two one-dimensional chains. Such a partitioning not only simplifies the analysis, but also significantly reduces the number of state variables.
\end{abstract}

\section{INTRODUCTION}

In [5], Bianchi presented a two-dimensional discrete time Markov chain to study the performance of IEEE 802.11 Distributed Coordination Function (DCF) under saturation conditions. The Markov chain depicted the behavior of backoff counter and backoff stage processes for a wireless station under the assumption of ideal channel conditions. Ziouva and Theodore [3] modified Bianchi's model by accounting for busy medium conditions. Subsequent studies extended either Bianchi's or Ziouva and Theodore's work to evaluate the performance of: (i) DCF under non-saturation conditions [4], (ii) $\mathrm{DCF}$ with finite retry limits ([6]), and (iii) quality of service (QoS) enhancements for DCF ([8], [9], [10]). In this document, we claim that the two-dimensional chain originally proposed by Bianchi or Ziouva and Theodore can be partitioned into two one-dimensional chains. Using the models presented in [3] and [6] as a framework, we build a twodimensional discrete time Markov chain depicting the behavior of a station operating under saturation conditions. We account for: (i) busy medium conditions when the backoff counter is frozen when the channel is busy, and (ii) finite retry limits $(R)$. We partition such a two-dimensional model into two one-dimensional chains, one depicting the behavior of backoff counter process and the other illustrating the behavior of retry counter process. Any extensions proposed to the original twodimensional model can also be directly applied to the resulting one-dimensional model. Such a partitioning simplifies the analysis of more complicated extensions to DCF scheme by significantly reducing the number of state variables.

\section{TWO-DIMENSIONAL MODEL}

Fig. 1 illustrates the two-dimensional Markov chain model depicting the behavior of backoff and retry counter processes for an arbitrary station, say $A$, under saturation conditions.
As seen from the figure, the model takes into account busy medium conditions [3] and assumes finite retry limits [6]. DCF employs a binary exponential backoff scheme. At each transmission attempt, the backoff counter is initialized to a random value uniformly chosen in the range $[0 \ldots C W)$, where $C W$ stands for the current contention window size. The value of $C W$ depends on the number of failed transmission attempts experienced by the head-of-line frame (i.e. the value of retry counter). For the very first transmission attempt, retry counter is zero and $C W$ is set equal to the minimum contention window size, $W_{0}$. After each unsuccessful transmission, the value of retry counter is incremented by one and $C W$ is doubled up to a maximum value of $W_{m}$, where $W_{m}=2^{m} W_{0}$. Following $m$ retransmission attempts, the backoff counter is initialized in the range $\left[0 \ldots W_{m}\right)$ after each subsequent failed transmission. Upon successful transmission or when the retry limit is reached, the head-of-line frame is discarded and the value of $C W$ is reset to $W_{0}$. Throughout this work, we assume that $m \leq R$ [6]. Suppose $i$ denotes the number of retransmission attempts experienced by the head-of-line frame, then the following relation for contention window size is valid:

$$
W_{i}=\left\{\begin{array}{l}
2^{i} \times W_{0}, \text { if } 0 \leq i \leq m \\
2^{m} \times W_{0}, \text { if } m<i \leq R
\end{array}\right.
$$

\section{ONE-DIMENSIONAL MODEL}

There are a total of $R+1$ rows in the two-dimensional discrete time Markov chain shown in fig 1 . The number of states in each row doubles as we move from row 0 to row $m$, and thereafter, remains the same. Let $b_{i, k}$ be the steady state probability that the retry and backoff counters of station $A$ are equal to $i$ and $k$, respectively. The probability conservation relation for the Markov chain can be written as:

$$
\begin{gathered}
\sum_{k=0}^{W_{m}-1} \sum_{i=0}^{R} b_{i, k}=1 \wedge b_{i, k}=0 \text { if } k \geq W_{i} \\
\Rightarrow \sum_{k=0}^{W_{m}-1} \sum_{i=m^{\prime}}^{R} b_{i, k}=1, \text { where } m^{\prime}=\left\lceil\log _{2}\left(\left\lceil\frac{k+1}{W_{0}}\right\rceil\right)\right\rceil
\end{gathered}
$$

In the above equation, $m^{\prime}$ is the first row between 0 and $R$ such that the number of states in $m^{\prime}\left(=2^{m^{\prime}} \times W_{0}\right)$ is greater than $k$. The significance of using $m^{\prime}$ in the above summation 


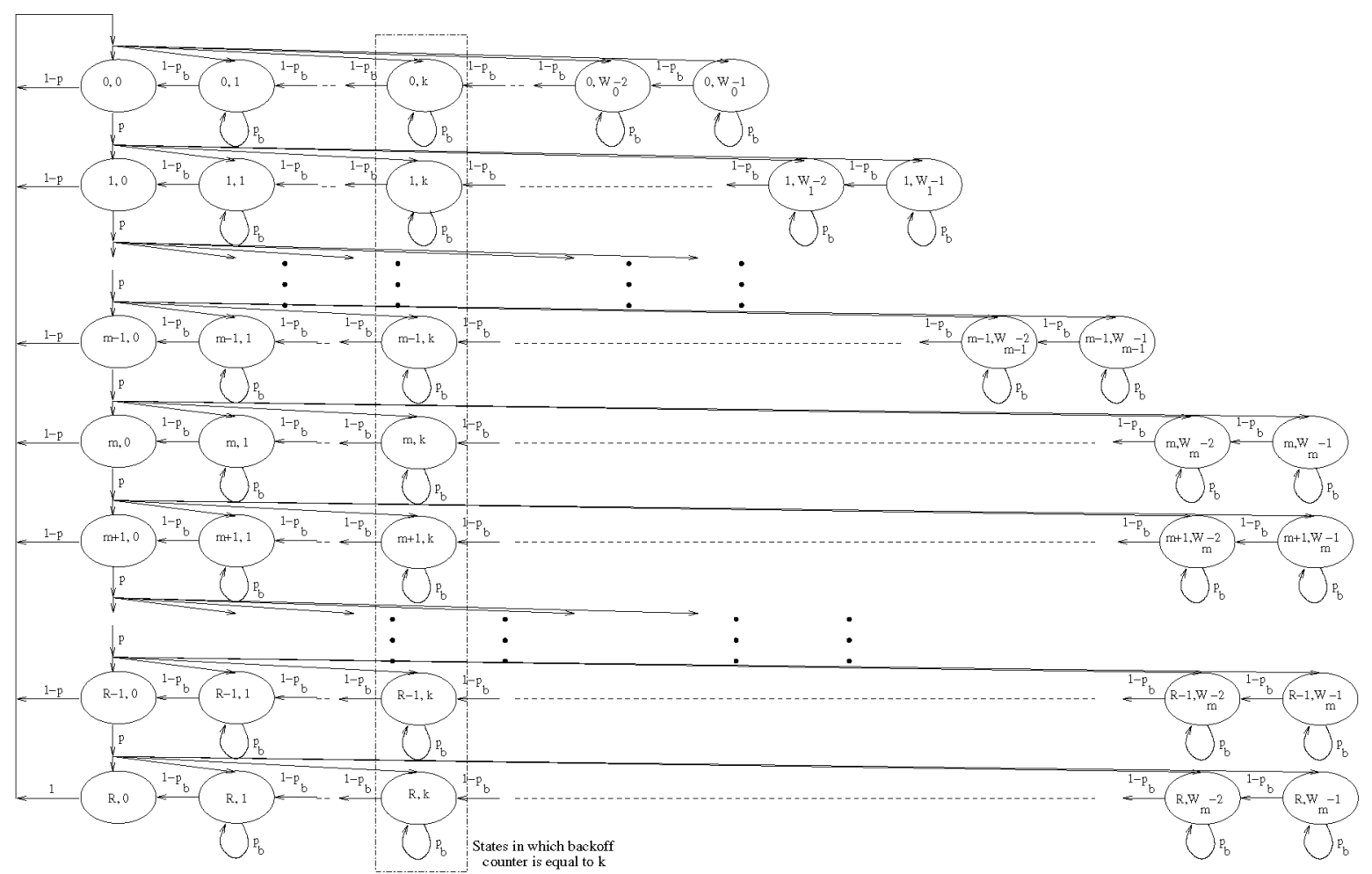

Fig. 1. Two-dimensional Markov chain model depicting the behavior of a wireless station

is that for all rows $j<m^{\prime}$, there is no state corresponding to $\{j, k\}$. Let $\mathcal{B}_{k}$ be the steady state probability that the considered station $A$ has backoff counter equal to $k$. Thus, $\mathcal{B}_{k}=\sum_{i=m^{\prime}}^{R} b_{i, k}$ as shown in fig. 1 by a dotted rectangle. Note that $\sum_{k=0}^{W_{m}-1} \mathcal{B}_{k}=1$. Let $n$ be the total number of stations in the network. Let $E_{k}$ be defined as the probability that all other $n-1$ stations in the network (i.e. stations other than station $A$ ) have backoff counter greater than or equal to $k$. Thus, $E_{k}=\left(\sum_{j=k}^{W_{m}-1} \mathcal{B}_{j}\right)^{n-1}$. Busy probability, $p_{b}$, is the probability that station $A$ senses the medium to be busy. This is same as the probability that at least one of the other $n-1$ stations transmit. Since, stations transmit only when their backoff counter is zero, $p_{b}=1-E_{1}$. Conditional collision probability, $p$, is defined as the probability that a frame transmitted by station $A$ experiences a collision. Since, $E_{0}-E_{1}$ is the probability that at least one of the other $n-1$ stations has backoff counter exactly equal to zero, $p=E_{0}-E_{1}=1-E_{1}$.

Under steady state, the probability of entering a state is equal to the probability of leaving the state. First, consider the states in row 0 . It is possible to enter a state, say $\{0, k\}$, when one of the following mutually exclusive events occur during a slot:

(i) Upon successful transmission or when the retry limit is reached, the station initializes its backoff counter to a value in the range $\left[0, W_{0}\right)$. Thus, it is possible to enter state $\{0, k\}$ after a transmission with a probability equal to $\frac{\substack{R-1 \\ i=0}_{b i, 0}(1-p)+b_{R, 0}}{W_{0}}$.

(ii) When $k>0$, the station freezes its backoff counter if the channel is sensed busy during a slot. This occurs with probability $p_{b}$.

(iii) The station enters state $k$ from state $k+1$ by decrementing its backoff counter by one after sensing the channel idle during a slot. This event occurs with probability $1-p_{b}$.

Thus, for retry counter $i=0$, we get:

$$
b_{0, k}=\left\{\begin{array}{c}
\frac{\int_{i=0}^{R-1} b_{i, 0}(1-p)+b_{R, 0}}{W_{0}}+b_{0, k+1}\left(1-p_{b}\right), \text { if } k=0 \\
\frac{R-1}{i=0} b_{i, 0}(1-p)+b_{R, 0} \\
W_{0} \\
+b_{0, k+1}\left(1-p_{b}\right), \text { if } 0<k<W_{0}
\end{array}\right.
$$

Now, consider the states in non-zero rows, $0<i \leq R$. Once again, it is possible to enter a state, say $\{i, k\}$, when one of the above-mentioned mutually exclusive events occur during a slot with one subtle variation. The station enters the states in row $i>0$ only when its $i^{\text {th }}$ transmission attempt fails. Thus, we have:

$b_{i, k}=\left\{\begin{array}{l}\frac{b_{i-1,0} \times p}{W_{i}}+b_{i, k+1}\left(1-p_{b}\right), \text { if } k=0 \\ \frac{b_{i-1,0} \times p}{W_{i}}+b_{i, k} p_{b}+b_{i, k+1}\left(1-p_{b}\right), \text { if } 0<k<W_{i}\end{array}\right.$

From equation (2), we have:

$$
\begin{aligned}
b_{i, W_{i}-1}=\frac{b_{i-1,0} \times p}{W_{i}} & +b_{i, W_{i}-1} p_{b}, \quad \because b_{i, W_{i}}=0 \\
\Rightarrow b_{i, W_{i}-1}\left(1-p_{b}\right) & =\frac{b_{i-1,0} \times p}{W_{i}} \\
& =\frac{W_{i}-\left(W_{i}-1\right)}{W_{i}} b_{i-1,0} \times p
\end{aligned}
$$




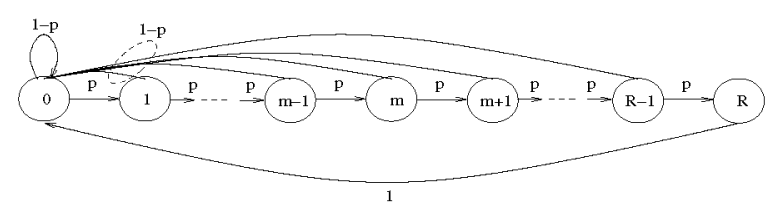

(a) Chain for retry counter process

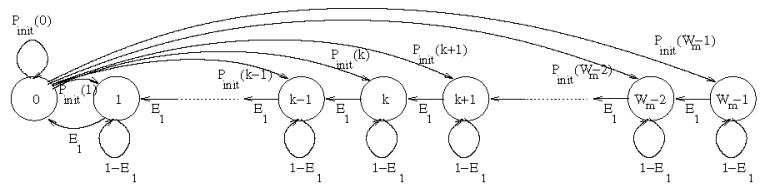

(b) Chain for backoff counter process

Fig. 2. One-dimensional chains

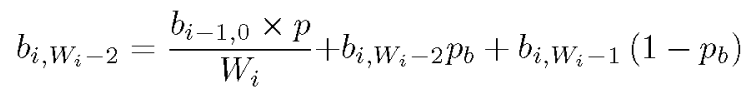

$$
\begin{aligned}
& \Rightarrow b_{i, W_{2}-1}\left(1-p_{b}\right)=\frac{b_{i-1,0} \times p}{W_{i}}+b_{i, W_{i}-1}\left(1-p_{b}\right) \\
& =\frac{b_{i-1,0} \times p}{W_{i}}+\frac{b_{i-1,0} \times p}{W_{i}} \\
& =\frac{2}{W_{i}} b_{i-1,0} \times p \\
& =\frac{W_{i}-\left(W_{i}-2\right)}{W_{i}} b_{i-1,0} \times p
\end{aligned}
$$

Generalizing, we have:

$$
b_{i, k}\left(1-p_{b}\right)=\frac{W_{i}-k}{W_{i}} b_{i-1,0} \times p, 0<k<W_{i}
$$

Finally, when $k=0$,

$$
\begin{aligned}
b_{i, 0} & =\frac{b_{i-1,0} \times p}{W_{i}}+b_{i, 1}\left(1-p_{b}\right) \\
& =\frac{b_{i-1,0} \times p}{W_{i}}+\frac{W_{i}-1}{W_{i}} b_{i-1,0} \times p(\text { from equation }(3)) \\
& =b_{i-1,0} \times p
\end{aligned}
$$

Generalizing equation (4), we get:

$$
\begin{aligned}
b_{i, 0} & =p^{i} \times b_{0,0}, 0 \leq i \leq R \\
\therefore \mathcal{B}_{0} & =\sum_{i=0}^{R} b_{i, 0}=b_{0,0} \frac{1-p^{R+1}}{1-p} \\
\Rightarrow b_{0,0} & =\mathcal{B}_{0} \frac{1-p}{1-p^{R+1}}
\end{aligned}
$$

Using (4) and (5), equation (1) can be expressed as follows:

$$
\begin{aligned}
& b_{0, k}=\left\{\begin{array}{c}
\frac{c_{i=0}^{R-1} p^{i} \times b_{0,0}(1-p)+p^{R} b_{0,0}}{W_{0}}+b_{0, k+1}\left(1-p_{b}\right), \text { if } k=0 \\
\frac{R-1}{i=0} p^{i} \times b_{0,0}(1-p)+p^{R} b_{0,0} \\
W_{0} \\
+b_{0, k+1}\left(1-b_{0}\right), \text { if } 0<k<W_{0}
\end{array}\right. \\
& =\left\{\begin{array}{l}
\frac{b_{0,0}}{W_{0}}+b_{0, k+1}\left(1-p_{b}\right), \text { if } k=0 \\
\frac{b_{0,0}}{W_{0}}+b_{0, k} p_{b}+b_{0, k+1}\left(1-p_{b}\right), \text { if } 0<k<W_{0}
\end{array}\right.
\end{aligned}
$$

Similarly, using (4) and (5), equation (2) can be simplified as follows:

$$
b_{i, k}=\left\{\begin{array}{l}
\frac{b_{i, 0}}{W_{i}}+b_{i, k+1}\left(1-p_{b}\right), \text { if } k=0 \\
\frac{b_{i, 0}}{W_{i}}+b_{i, k} p_{b}+b_{i, k+1}\left(1-p_{b}\right), \text { if } 0<k<W_{i}
\end{array}\right.
$$

Equations (7) and (8) can be together expressed as:

$$
b_{i, k}=\left\{\begin{array}{l}
\frac{b_{i, 0}}{W_{i}}+b_{i, k+1}\left(1-p_{b}\right), \text { if } k=0 \\
\frac{b_{i, 0}}{W_{i}}+b_{i, k} p_{b}+b_{i, k+1}\left(1-p_{b}\right), \text { if } 0<k<W_{i}
\end{array}\right.
$$

Taking summation over $i$ on both sides, we get:

$\mathcal{B}_{k}=\left\{\begin{array}{l}\sum_{i=m^{\prime}}^{R} \frac{b_{i, 0}}{W_{i}}+\mathcal{B}_{k+1} E_{1}, \text { if } k=0 \\ \sum_{i=m^{\prime}}^{R} \frac{b_{i, 0}}{W_{i}}+\mathcal{B}_{k}\left(1-E_{1}\right)+\mathcal{B}_{k+1} E_{1}, \text { if } 0<k<W_{m}\end{array}\right.$

In equation (9), the term $\sum_{i=m^{\prime}}^{R} \frac{b_{i, 0}}{W_{i}}$ can be expressed in the following two ways:

(i) Using equations (5) and (6),

$$
\sum_{i=m^{\prime}}^{R} \frac{b_{i, 0}}{W_{i}}=b_{0,0} \sum_{i=m^{\prime}}^{R} \frac{p^{i}}{W_{i}}=\mathcal{B}_{0} \sum_{i=m^{\prime}}^{R} \frac{(1-p) p^{i}}{\left(1-p^{R+1}\right) W_{i}}
$$

(ii) By definition, $b_{i, 0}$ is the steady state probability that a station whose head-of-line frame has been transmitted $i$ times, transmits in a slot. Thus, $b_{i, 0}=\operatorname{Prob} .\{r=i \cap$ $t x\}=\operatorname{Prob} .\{t x\} \times \operatorname{Prob} .\{r=i \mid t x\}$. Here, Prob. $\{t x\}$ is the (unconditional) probability that a station transmits during a slot and Prob. $\{r=i \mid t x\}$ is the probability that a transmitting station has retry counter equal to $i$. Thus,

$$
\sum_{i=m^{\prime}}^{R} \frac{b_{i, 0}}{W_{i}}=\operatorname{Prob} .\{t x\} \sum_{i=m^{\prime}}^{R} \frac{\operatorname{Prob} .\{r=i \mid t x\}}{W_{i}}
$$

From equations (10) and (11), it is clear that Prob. $\{t x\}=$ $\mathcal{B}_{0}$ and Prob. $\{r=i \mid t x\}=\frac{(1-p) p^{i}}{\left(1-p^{R+1}\right)}$. A formal way to derive expression for Prob. $\{r=i \mid t x\}$ is to compute the steady state probability distribution from a discrete time one-dimensional chain of the retry counter process (as shown in fig. 2(a)). The transitions in the chain take place at the beginning of a slot immediately following a transmission by the considered station.

Let $P_{\text {init }}\left(k, W_{i}\right)$ be the probability of initializing backoff counter equal to $k$ when the contention window size is $W_{i}$. Thus, $P_{\text {init }}\left(k, W_{i}\right)=\frac{1}{W_{i}}$. Let $P_{\text {init }}(k)=\sum_{i=m^{\prime}}^{R} \operatorname{Prob} .\{r=$ $i \mid t x\} P_{\text {init }}\left(k, W_{i}\right)$, which gives the steady state probability that the station initializes its backoff counter to $k$ after a generic transmission. Then, equation (9) can be expressed as:

$$
\mathcal{B}_{k}=\left\{\begin{array}{r}
\operatorname{Prob} .\{t x\} P_{\text {init }}(k)+\mathcal{B}_{k+1} E_{1}, \text { if } k=0 \\
\operatorname{Prob} .\{t x\} P_{\text {init }}(k)+\mathcal{B}_{k}\left(1-E_{1}\right) \\
+\mathcal{B}_{k+1} E_{1}, \text { if } 0<k<W_{m}
\end{array}\right.
$$

A formal way to derive equation (12) is to compute the steady state probability distribution of a discrete time one-dimensional chain depicting the behavior of the backoff counter process (as shown in fig. 2(b)). The transitions in the chain take place at the beginning of a slot. Thus, equation (12) denotes the global balance equation for a state $k$, where 
$0 \leq k<W_{m}$. The left hand side of the equation gives the probability of leaving state $k$. The right hand side of the equation represents the probability of entering state $k$. A station enters state $k$ when one of the following two transitions occur at the end of a slot:

- Post-transmission transition. This corresponds to a transition from state 0 to state $k$ when the station has just completed its transmission and re-initialized its backoff counter to $k$. This takes place with probability $P_{\text {init }}(k)$.

- Non-transmission transition. This corresponds to either a transition from state $k+1$ to state $k$ when the medium is sensed idle or a transition from state $k$ to itself when the medium is sensed busy during a slot. The former transition takes place with probability $E_{1}$ and the latter with probability $1-E_{1}$.

Note that the non-transmission transition from a state $k$ to itself holds good only for $k>0$.

\section{DISCUSSION}

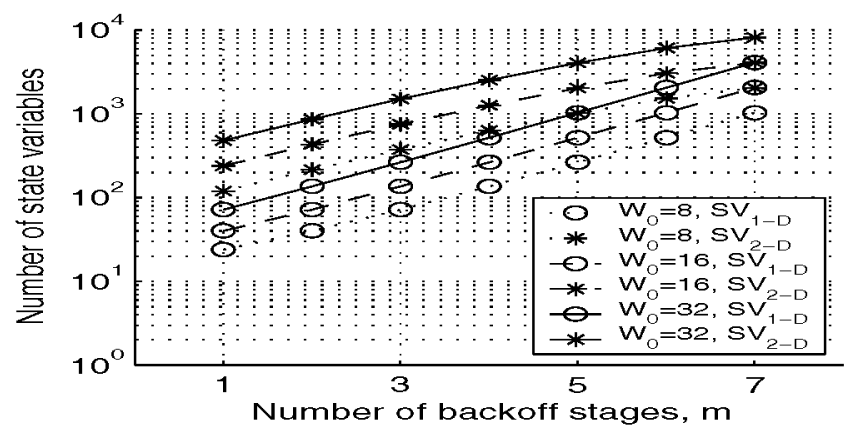

Fig. 3. Number of state variables with $R=7$

Let $S V_{2-D}$ denote the number of state variables in the twodimensional Markov chain shown in fig. 1. Then,

$$
\begin{aligned}
S V_{2-D} & =W_{0}\left(2^{0}+2^{1}+2^{2}+\ldots+2^{m-1}\right)+(R-m+1) W_{m} \\
& =(R-m+2) W_{m}-W_{0}
\end{aligned}
$$

On the other hand, the two one-dimensional chains shown in fig. 2 have a total of $S V_{1-D}=W_{m}+R+1$ states. Fig. 3 compares the number of state variables that need to be maintained by employing one two-dimensional chain and two onedimensional chains. When Bianchi's or Ziouva and Theodore's Markov chain models are used for a more complicated analysis like evaluating the effectiveness of QoS enhancements for DCF, we need to solve for each state variable using a nonlinear equation [9]. Hence, in order to obtain the steady state probabilities for backoff and retry counter, a system of nonlinear equations needs to be solved. It is desirable to reduce the number of state variables for the following reasons:

1) Convergence rate of methods to solve the system of non-linear equations (for example, fixed-point iteration, bisection method, Newton-Raphson) largely depend on the initial guessed values. Greater the number of state variables, greater is the difficulty in making a "good" guess for initial values [2]. For example, if the initial value is too far from the true solution, NewtonRaphson's method can fail to converge.

2) Greater the number of state variables, larger is the system of non-linear equations that needs to be solved. This increases the time taken to converge to the correct solution. For example, computational complexity of NewtonRaphson method in $N$-dimensions is $O\left(N^{3}\right)$ [7].

Note that equation (12) is derived directly from the steady state expressions corresponding to the two-dimensional Markov chain in fig. 1. Hence, any extensions to the twodimensional models presented in [3] and [5] can also be applied to the one-dimensional chains. For example, the two one-dimensional chains shown in fig. 2 can be used to evaluate the performance of DCF scheme with finite retry limits. QoS enhancements to DCF scheme employ different values for minimum/maximum contention window for each traffic class or access category [1]. The effectiveness of such enhancements can be studied by substituting appropriate values for $m, W_{0}$ or $W_{m}$ and using the one-dimensional chains in fig. 2 to illustrate the behavior of an access category within a station.

\section{REFERENCES}

[1] Draft supplement to Part 11 - Wireless Medium Access Control (MAC) and Physical (PHY) Layer Specifications: MAC enhancements for Quality of Service (QOS), IEEE P802.11e/D9.0, August 2004.

[2] D. C. McKinney. Numerical methods for civil engineers. In Lecture notes on non-linear equations, Department of Civil Engineering, University of Texas at Austin, October 2004.

[3] E. Ziouva and T. Antonakopoulos. CSMA/CA performance under high traffic conditions: throughput and delay analysis. In Computer Communications, pages 313-321, February 2002.

[4] F. Alizadeh-Shabdiz and S. Subramaniam. A finite load analaytical model for the IEEE 802.11 Distributed Coordinated Function MAC. In Proceedings of WiOpt, pages 321-322, March 2003.

[5] G. Bianchi. Performance analysis of the IEEE 802.11 Distributed Coordination Function. In IEEE JSAC, pages 535-547, March 2000.

[6] H. Wu, Y. Peng, K. Long, S. Cheng and J. Ma. Performance of reliable transport protocol over IEEE 802.11 wireless LAN: analysis and enhancement. In IEEE Infocom, pages 599-607, June 2002.

[7] W. H. Press et al. Numerical recipes in C, The Art of Scientific Computing, Second Edition, 1992.

[8] Y. Xiao. An analysis for differentiated services in IEEE 802.11 and IEEE 802.11e Wireless LANs. In IEEE ICDCS, pages 32-39, March 2004.

[9] Z. Tao and S. Panwar. An analytical model for IEEE 802.11e Enhanced Distributed Coordination Function. In IEEE ICC, June 2004.

[10] Zhen-ning Kong, Danny H. K. Tsang, Brahim Bensaou and Deyun Gao. Performance analysis of IEEE 802.11e contention-based channel access. In IEEE Journal on Selected Areas in Communications, pages 917-928, December 2004. 